\title{
La hermenéutica como corriente aplicada al campo de la educación de la enfermería
}

\section{Hermeneutics Applied to the Field of Nursing Education}

\author{
Elena Mora-Escalante ${ }^{1}$ \\ Universidad de Costa Rica \\ Escuela de Enfermería \\ Docente Asociado \\ San José, Costa Rica \\ moresca21@hotmail.com
}

Recibido: 7 agosto 2013 Aceptado: 4 diciembre 2014 Corregido: 28 agosto 2014

Resumen: El artículo científico titulado la hermenéutica como corriente aplicada al campo de la educación de la enfermería, tiene como fin reflexionar sobre el reto que representa para el profesorado de la Escuela de Enfermería de la Universidad de Costa Rica mediar la propuesta curricular acorde con el paradigma asumido. Se plantea la forma de entender el proceso formativo desde el paradigma hermenéutico, donde la educación es comprendida como un proceso íntimo de formación de la persona desde una perspectiva holistica. Se propone una pedagogía abierta a la experiencia, vivencia y comprensión de los sujetos, los cuales son los actores y el centro del proceso y que como sujeto histórico, subjetivo, inter e intra-subjetivo, busca el diálogo para construir el conocimiento que se gesta en lo social y es asimilado y transformado en lo individual. Esto implica un compromiso del profesorado para comprender el fundamento paradigmático de la propuesta curricular y desarrollar una pedagogía hermenéutica.

Palabras clave: teoría educativa, corriente hermenéutica, pedagogía hermenéutica, enfermería.

Abstract: The scientific article entitled the hermeneutics applied to the branch of nursing education, it intend to reflect about the challenge posed to the faculty of the School of Nursing at the University of Costa Rica. It was focused on mediating curricular proposal in line with the assumed paradigm. Arises how to understand the training process from hermeneutics, where education is understood as a formation process of the individual from a holistic perspective. It is proposed a pedagogy open to experience and understanding of the subjects whom are actors and the center of the process and as an historical subject, subjective, inter-and intra-subjective seeks dialogue to construct knowledge that is brewing in social and is assimilated and transformed individually. This involves a commitment of teachers to understand the paradigmatic behavior of the curriculum proposal and develop a hermeneutic pedagogy.

Keywords: educational theory, hermeneutics, hermeneutic pedagogy, nursing.

"me preocupa la creciente distancia entre la práctica educativa y el ejercicio de la curiosidad epistemológica"

(Freire, 1995).

1 Profesora Adjunta de la Universidad de Costa Rica. Enfermera. Licenciada en Salud Mental y Psiquiatría. Master en Terapia Familiar Sistémica. 


\section{Introducción}

A través del presente artículo científico, se expone el cambio de paradigma realizado por la Escuela de Enfermería de la Universidad de Costa Rica, su repercusión en la forma de enseñar y aprender el objeto de estudio, así como el desafío que debe de enfrentar el/la docente para mediar la propuesta curricular de forma congruente con lo estipulado.

Inicialmente se presenta la posición paradigmática, epistemológica y pedagógica que sustenta el currículo de la carrera de Licenciatura en Enfermería de la Universidad de Costa Rica y posteriormente, se analiza el aporte de la teoría del conocimiento en el campo de la educación, en el ámbito teórico y en relación con la práctica educativa.

Los fundamentos se extraen de investigaciones, revisiones y publicaciones recientes relacionadas con la temática.

\section{Desarrollo}

La Escuela de Enfermería de la Universidad de Costa Rica en el año de 1997, realizó la reestructuración de su Plan de Estudios de Bachillerato con el objetivo de crear un plan de estudio con una mayor vigencia de cara al futuro y que a la vez, le permita a la enfermería reconocerse como una disciplina distinta de la disciplina médica, con una formación que faculte realizar cuidados de enfermería acordes con los procesos, los problemas y situaciones de las personas de todas las edades, en todas las situaciones de la vida y en un mundo en constante evolución. (Comisión de Currículum, 1997).

Por lo anterior, se analizó la posición paradigmática que debía asumir la Escuela, que responda de forma congruente con esta necesidad y que permita plantearse una nueva experiencia, orientada a una transformación con implicaciones en la enseñanza, la investigación y la práctica de enfermería, ya que el paradigma, según Fourez (2008), actúa como una norma para decidir lo que se va estudiar y de qué manera; representa una matriz disciplinar que contiene valores, reglas, reglamentos y métodos de construcción del conocimientos.

De esta manera, la Escuela de Enfermería asume el paradigma interpretativohermenéutico, el cual se enfoca en la comprensión, el significado y la acción del mosaico social. Sus designios primordiales están encaminados a la comprensión de la conducta humana a través del develamiento de los significados sociales (Comisión de Currículum, 1997).

La hermenéutica en su sentido más tradicional, es la ciencia y el arte de interpretar, es ciencia porque contiene un conjunto de principios y reflexiones teóricas en torno a la interpretación y es técnica, porque regula la forma en que se ha de interpretar algo. Es la aceptación de la idea fundamental que todo nuestro conocimiento del mundo está mediado de cierta manera por la interpretación (Monzón, 2011).

Por otra parte, la hermenéutica necesariamente se relaciona con el saber práctico en el que supera la simple reflexión teórica y se traduce en actuaciones concretas. En definitiva, en eso estriba la hermenéutica filosófica: "en que todo comprender es siempre aplicar; la interpretación y comprensión, siempre han de estar en horizonte de aplicación” (Gadamer, 2001). 
La hermenéutica como corriente aplicada al campo de la educación de la enfermería cuyo objeto de estudio es el cuidado, concuerda perfectamente, ya que la práctica de los cuidados de salud es primariamente una relación entre personas y para comprender esa práctica del cuidado, se requiere del entendimiento de los significados e intenciones presentes en las relaciones entre personas. Por lo tanto, las relaciones entre el/la estudiante de enfermería y la persona cuidada, son también captadas en términos de su significado para ambas. En estos casos es cuando interviene la hermenéutica, para buscar la comprensión además del entendimiento (Beuchot, 2007).

En conjunto con la posición paradigmática, se definió como sujeto de conocimiento al estudiante y como objeto de conocimiento el cuidado de la salud - enfermedad humana.

En este sentido, el cuidado es considerado como la esencia, la unificación intelectual y las dimensiones prácticas de la profesión, abordado en dos líneas indisociables: Una es la relacionada propiamente con la práctica clínica y la otra, se puede ubicar en la interacción socio afectiva que significa estar juntos, desmitificando la relación de poder entre la enfermería y el individuo, de esta manera, el cuidado se constituye en una relación terapéutica en la que se concibe a la persona como un ser humano con un trasfondo sociocultural, una historia personal, valores, creencias y con derecho de participar en la toma de decisiones, lo que implica que las acciones deben ser determinadas en conjunto. Así la esencia de enfermería es el cuidado de las personas en congruencia con su cultura, valores y patrones de vida (Comisión de Currículo, 1997).

Evidentemente el concepto de cuidado denota fenómenos complejos y multidimensionales, que posee elementos científico-técnicos (orientados a la dimensión biológica del enfermar), fenomenológicos y humanísticos (orientados a la esfera psicosocial y espiritual de la enfermedad).

Las características antes señaladas, tienen importantes repercusiones en la enseñanza y el aprendizaje del cuidado como objeto de estudio, y por lo tanto para el/la docente de enfermería. El/la docente debe comprender e interiorizar la propuesta curricular en su totalidad, para poder facilitarle al estudiante la construcción del significado del cuidado a través de la comprensión hermenéutica.

Relacionado a esta concepción de construcción del significado, la Escuela de Enfermería planteó que la enseñanza centrada en el cuidado no puede ser impartida bajo los parámetros tradicionales del enfoque positivista-tecnológico, donde se concibe el hecho educativo desde la premisa objetiva de relaciones de causa y efecto, al margen de los significados atribuidos por los sujetos, que desde la noción de ciencias de la naturaleza disgrega las relaciones entre sujetos, instituciones y acontecimientos y en donde se procede mediante la utilización de un método fundamentado en la observación, la experimentación, la generalización, la predicción y el rigor (Velázquez, 2005).

Por lo anterior, la Comisión de Currículum de la Escuela de Enfermería (1997), consideró que el enfoque fenomenológico-interpretativo, es el indicado para orientar y determinar la forma de concebir, conocer, organizar y proceder en el proceso de enseñanza-aprendizaje, ya que "el comportamiento humano solo puede ser interpretado por referencia a los motivos del actor, a sus intenciones y al contexto social dentro del cual adquieren sentido tales intenciones" (p. 35). 
Este enfoque señala que la educación es un fenómeno de interacción social, en el que los criterios de interpretación y comprensión son construidos, comunicados y enseñados por los sujetos, por lo que se procede desde métodos hermenéuticos y etnográficos (Velázquez, 2005).

Mientras que la didáctica según este enfoque, orienta la estrategia de los saberes específicos en el orden de la enseñanza; se entiende, como un conjunto de argumentaciones sobre la práctica del enseñar y del aprender en el aula, lo cual hace referencia a conceptos teóricos y conceptos operativos que impiden la asimilación de la didáctica a meras fórmulas (Zuluaga et al., 2003).

Representa concebir la educación como proceso social, como experiencia viva para los involucrados en los procesos y para las instituciones educativas, enfatiza que, transformando la conciencia de los docentes, estos transformarán su práctica educativa (Comisión de Currículum, 1997).

En congruencia con lo anterior, se establece asumir un modelo pedagógico, que brinde las pautas en las que discurrirá la formación, que proponga nociones, fundamentos y estrategias que consideran el contexto, las finalidades, los sujetos, los recursos económicos y materiales, el contenido y el tiempo (Arias, Francis y Marín, 2013).

El modelo pedagógico adoptado por la Escuela de Enfermería fue el constructivista, por ser un modelo pertinente para la formación del profesional de enfermería, en donde la tarea de educar no se reduce a transmitir información en forma de datos, fechas, fórmulas o hechos, en su lugar la educación busca la formación de los seres humanos.

Se parte de que la construcción del conocimiento se da en forma personal, particular, pero a partir de experiencias que se comparten con los demás. Este aprendizaje tiene su base en lo que cada quien ya sabe; es decir, que aunque se comparta una experiencia cada individuo aprende en forma particular en virtud de lo que ya sabe. "El aprendizaje se efectúa cuando la persona establece relaciones sustanciales entre los conceptos nuevos y aquellos que ya han sido incorporados en su estructura cognoscitiva" (Comisión de Currículum, 1997. p 45).

De esta forma, la propuesta curricular plantea una construcción del significado del cuidado a través del enfoque fenomenológico-interpretativo, donde se procede desde métodos hermenéuticos, por lo tanto, es trascendental que el/la docente de la Escuela de Enfermería realice una "Pedagogía Hermenéutica".

Luis Antonio Monzón (2011), en su artículo titulado La identidad docente desde una perspectiva hermenéutica, señala que la pedagogía hermenéutica, requiere "colocar las acciones educativas en un contexto determinado, tratar de darles sentido, tratar de entender y comprender este proceder y estas formas de actuar, tanto a nivel individual como a nivel colectivo; es considerar que no hay hechos pedagógicos, sino interpretación pedagógica de los hechos“ (p.7).

Ana Galvis (2009), menciona en su ensayo Pedagogía y Hermenéutica en el Aula de Clase que la hermenéutica es en el campo educativo un medio universal de apropiación de la realidad, una forma de acceder a las realidades humanas, haciendo lectura de la verdad de sus contextos:

La hermenéutica le permite al educador comprender que la realidad tiene gran sentido por su relación con los seres humanos sin distingo de edades, géneros, raza, etc. La hermenéutica 
permite al educador, interiorizar que la comprensión que tenemos del pasado, se debe gracias a que se produce una fusión de horizontes entre el pasado y el presente. La hermenéutica pasa de ser teórica para convertirse en algo que hay que hacer (p. 4).

Jordi Planella (2005), en el ensayo Pedagogía y Hermenéutica. Más allá de los datos en la educación, refuerzan la pertinencia que tiene este paradigma para la práctica docente en la Escuela de Enfermería:

Impregna la realidad pedagógica más allá de posiciones exclusivamente cuantificables, donde se entiende la educación como un proceso íntimo de formación del hombre desde una perspectiva holística, por lo que no propone un modelo pedagógico canónico, sino que lo propone abierto, a la misma vivencia y comprensión de los sujetos, reubicando el sujeto en el centro de la pedagogía más allá de la insistencia en la búsqueda de objetividad y parte de la propia experiencia, del reconocimiento del sujeto como actor, que como sujeto histórico busca el diálogo (p. 7).

Hans Georg Gadamer (2001), en el artículo titulado La educación es educarse, explica:

Un profesor es una persona que, por sus cualidades y conocimientos, ejercita una praxis fundada en sus conocimientos y para llevar a otros a que abran sus propios horizontes de comprensión frente a sí mismos y a la sociedad, a través de un corpus teórico y técnico determinado por la cultura. Estas acciones se viabilizan por medio de los procesos de enseñanza y aprendizaje. Allí hay un auténtico fenómeno hermenéutico: la labor de la hermenéutica es siempre esa transferencia desde un mundo a otro. Por lo que sí es posible una pedagogía hermenéutica, en la medida que la aplicación pedagógica pueda traspasar la simple reproducción de técnicas y se aproxime a los horizontes teleológicos de la sociedad y de la cultura, para producir aplicaciones concretas. Ser maestro hermeneuta o hacer pedagogía hermenéutica, finalmente, es pensar la educación desde las dimensiones humanas de la comprensión del sentido (p.42).

Por su parte, Guanipa y Velazco, (2005) en el artículo Hermenéutica del discurso pedagógico del profesor, establecen que el ambiente que se crea conlleva a generar diálogos inteligentes, donde la vivencia y experiencia del docente se mezcla con las vivencias de los estudiantes, fomentando así una discusión crítica donde el aprendizaje se torna efectivo (p. 7-8).

El asumir el paradigma hermenéutico en la formación de enfermeras(os) exige un cambio en la praxis pedagógica tradicional hacia una pedagogía hermenéutica, que conlleva que el profesorado conciba la educación como proceso social, como una experiencia viva para los involucrados y para las instituciones educativas, que entiende a la educación como un proceso íntimo de formación de la persona desde una perspectiva holística; para esto se requiere una transformación en su conciencia para que cambie su práctica educativa (Comisión de Currículum, 1997).

En términos prácticos, requiere que el profesorado revise la temática de tal forma que se logre una comprensión preliminar de la misma, valiéndose de las vivencias, experiencias y conceptos preconcebidos de sí misma(o) y del estudiantado, creando un ambiente que conduzca 
al dialogo para que se produzcan interacciones críticas, potenciando así las participación individual y grupal.

Posteriormente, acompaña al estudiante a un proceso de profundización y exploración de detalles sobre los contenidos analizados, a fin de consolidar los conocimientos. Para esto, desarrolla analogías, análisis crítico reflexivo y análisis comparativo, de tal manera que se logre una percepción lo más amplia posible del entorno que rodea la temática, propiciando la investigación, generando una apertura mental y una predisposición para la formulación de supuestos preliminares.

Completada esta etapa, realiza una exploración holística de la temática, lo que lleva a una comprensión profunda de la misma y para ello, guía al estudiante a generar sus propias interpretaciones de los contenidos a través de diálogos reflexivos, analizando la información de manera sistémica y así, llegue a conclusiones nuevas y productivas que puedan proyectarse en su entorno y en su futura práctica profesional (Guanipa y Velazco, 2005).

Además de lo anterior, el profesorado considera los significados que le atribuye el/la estudiante a sus vivencias, para que de esta manera identifique que significa estar en esa situación y lo significa para la persona a la que le brinda cuidado.

Promueve en el estudiantado la comprensión e interpretación de las vivencias de la persona respecto a su proceso de salud-enfermedad, considerando su trasfondo sociocultural, historia personal, valores, creencias, etc.; de esta manera, realicen un cuidado de enfermería comprensivo, interpretando e integrando lo que la persona expresa y percibe de su experiencia, lo que desea, necesita y espera del cuidado develado en un proceso de diálogo.

El objetivo es desarrollar un cuidado de las personas de manera integral, donde el Proceso de Atención de Enfermería se construye junto con la persona a la que le brindan cuidado, comprendiendo su modo de vivir y estableciendo una relación de respeto, aceptación, apoyo y acompañamiento.

\section{Consideraciones Finales}

Los programas de los módulos que se desarrollan en la Escuela de Enfermería reflejan concordancia entre metodología y actividades de aprendizaje con las premisas del paradigma hermenéutico, sin embargo, es necesario seguir insistiendo en una práctica docente acorde con lo estipulado y de esta forma poder sobrepasar la sola interpretación y comprensión de un fenómeno e ir a la aplicación.

Asumir este reto requiere un papel determinante del profesorado en el proceso de enseñanza-aprendizaje, se debe seguir creando espacios de reflexión en la Escuela de Enfermería que faciliten la construcción de la concepción del currículo, donde le quede claro a cada uno de las y los docentes lo que hace, cómo lo hace y para qué lo hace y de esta forma, se apropie del abordaje paradigmático, epistemológico y pedagógico propuesto y logre proyectarlo de forma coherente en su práctica docente.

Como lo menciona Carmen Trespalacios et al., (2012), 
no basta con desarrollar de forma mecánica una propuesta curricular, se demanda de la configuración de un sujeto-docente con conciencia, que pueda recuperar desde lo fenomenológico los sentidos y significados del acto pedagógico, que explore la urdimbre de las interacciones e intencionalidades curriculares, de manera que medie con su reflexión y su actuar, una formación encaminada a fortalecer la conciencia de los individuos en formación y a potenciar la configuración de sujetos sociales capaces de intervenir la realidad (p. 5).

\section{Referencias}

Arias, M., Francis, S., y Marín, P. (2013). Modelos pedagógicos de formación docente en la Universidad de Costa Rica. San José: Universidad de Costa Rica.

Beuchot, P. M. (2007). Hermenéutica analógica y educación. México: Calzada Iberoamericana.

Comisión de Currículum, Escuela de Enfermería. (Diciembre 1997). Reestructuración del bachillerato que se transforma en la licenciatura en enfermería resolución ( $n^{\circ}$ 6479-98). Universidad de Costa Rica.

Freire, P. (1995). A sombra desta mangueira. São Paulo: Olho d’Água.

Fourez, G. (2008). Cómo se elabora el conocimiento. Madrid: Editorial NARCEA.

Gadamer, H. G. (2001). La educación es educarse. Barcelona: Editorial Paidós.

Galvis, A. A. (14 de diciembre 2009). Pedagogía y Hermenéutica en el Aula de Clase. [Blogspot]. Recuperado de http://anabelsigalvis.blogspot.com/2009/12/pedagogia-y-hermeneutica-enel-aula-de.html

Guanipa, M.J. y Velazco, L. (2005) Hermenéutica del discurso pedagógico del profesor. Maracaibo. Revista Lingua Americana. 17, 130-150. Recuperado de http://www. highbeam.com/doc/1P3-1259027471.html

Monzón, L. A. (2011). La identidad docente desde una perspectiva hermenéutica. Revista de Educación y Desarrollo 18, 28-30. Recuperado de http://www.imbiomed.com/1/1/articulos. php? method=showDetail\&id_articulo=75007\&id_seccion=4504\&id_ejemplar=7477\&id_ revista $=291$ 
Planella, J. (2005). La identidad docente desde una perspectiva hermenéutica. Revista Iberoamericana de Educación 36(12).7-9. Recuperado de http://www.academia. edu/5885738/Revista_Iberoamericana_de_Educaci\%C3\%B3n_ISSN_1681-5653

Trespalacios Ortiz C., Rada Espinosa M., Hernández Flores M., Guerrero Hernández T., Landeros López M.. (2008). Curriculum y docente: encuentro de significados. Educación Médica Superior [revista en la Internet]. 22(2), 1-6 Recuperado de http://scielo.sld.cu/ pdf/ems/v22n2/ems05208.pdf

Velázquez, I. (2005). Una aproximación al mapa disciplinar de la pedagogía. Revista Iberoamericana de Educación 35(2), 9-10. Recuperado de http://www.rieoei.org/ deloslectores/811Velazquez.PDF

Zuluaga, O., Echeverri, A., Martínez, A., Quiceno, A., Sáenz, J. y Álvarez, A. (2003). Pedagogía y epistemología. Bogotá: Cooperativa editorial magisterio. 